\title{
Is late treatment with acetaminophen safe and effective in avoiding surgical ligation among extremely preterm neonates with persistent patent ductus arteriosus?
}

\author{
Sally Mashally ${ }^{1}$, Rudaina Banihani ${ }^{1,2}$, Bonny Jasani ${ }^{2,3}$, Lynne E. Nield ${ }^{1,2,3}$, Fernando F. Martins ${ }^{4}$, Amish Jain ${ }^{2,5}$ and Dany E. Weisz $\mathbb{D}^{1,2}{ }^{凶}$
}

(c) The Author(s), under exclusive licence to Springer Nature America, Inc. 2021

\begin{abstract}
OBJECTIVE: Evaluate the association of late treatment with acetaminophen vs. immediate surgical ligation with death or neurodevelopmental impairment (NDI) among extremely low gestational age neonates (ELGANs) with persistent patent ductus arteriosus (pPDA).

STUDY DESIGN: Retrospective comparative epoch study of ELGANs with pPDA being considered for surgical ligation. ELGANs in epoch 1 (2009-2012) were referred for ligation, while infants in epoch 2 (2012-2015) were treated with oral acetaminophen and referred for ligation in the absence of improvement. The primary outcome was a composite of death/NDI at 18-24 months. RESULTS: Ninety-two ELGANs with median[IQR] GA 25.2 weeks [24.4, 26.3] had pPDA (43 in epoch 1, 49 in -epoch 2) with acetaminophen-exposed neonates receiving 7 days $[7,7]$ of treatment. ELGANs in epoch 2 had reduced ligation (aOR 0.30; 95\%Cl: $[0.11,0.87])$, but there was no difference in death/NDI (aOR 1.03; 95\%Cl: $[0.30,3.56]$ ).

CONCLUSIONS: Late treatment with acetaminophen to avoid surgery for pPDA is associated with reduced ligation but no difference in death/NDI, supporting the safety and effectiveness of this approach.
\end{abstract}

Journal of Perinatology (2021) 41:2519-2525; https://doi.org/10.1038/s41372-021-01194-4

\section{INTRODUCTION}

The management of patent ductus arteriosus (PDA), which occurs in up to $60 \%$ of infants born extremely preterm [1], is an area of significant controversy. Persistent PDA after treatment with cyclooxygenase inhibitors (COXI) has been associated with increased mortality and neonatal morbidity [2]. However, methods of definitive ductal closure, such as surgical ligation, have been associated with adverse outcomes, including neurodevelopmental impairment (NDI) in early childhood [3-5], culminating in a trend of clinicians avoiding surgical PDA treatment over the past decade [6-9]. Late treatment with acetaminophen is an effective alternative to facilitate closure of persistent PDA [10] and avoid surgical ligation among extremely preterm neonates [11]. With no demonstrated early adverse effects or neonatal outcomes associated with acetaminophen treatment, neonatologists are now commonly prescribing acetaminophen for PDA, with over $25 \%$ of extremely preterm infants with PDA receiving treatment with acetaminophen [12].

There remains, however, significant uncertainty regarding the long-term safety of administering acetaminophen for PDA treatment, including among ELGANs being considered for surgical ligation, among whom over half are subsequently diagnosed with neurodevelopmental impairment in early childhood [13, 14]. Foetal exposure to acetaminophen has been associated with increased language delay, attention deficit hyperactivity disorder
(ADHD) and autism spectrum disorder (ASD) [15-18]. While the administration of short courses of acetaminophen for analgesia [19], PDA prophylaxis [20] or very early PDA treatment [21] among neonates has not been associated with NDI, no studies have evaluated the association of later or prolonged courses of acetaminophen treatment for symptomatic PDA with neurodevelopmental outcomes. Developmental evaluation in early childhood has been recommended for preterm neonates treated with acetaminophen for PDA in light of concerns regarding the risk of NDI following postnatal exposure [22].

The objective of this study was to evaluate the association of late acetaminophen treatment (with surgical ligation 'back-up') vs. immediate ligation for persistent large PDA with death or neurodevelopmental impairment in early childhood.

\begin{abstract}
METHODS
We conducted a retrospective comparative epoch cohort study of extremely low gestational age neonates (ELGANs, born at gestational age $[G A] \leq 28$ weeks) with persistent large PDA being considered for surgical ligation after failure of COXI and/or conservative therapy. The neonatal intensive care unit at Sunnybrook Health Sciences Centre in Toronto, Canada is a tertiary perinatal centre that admits approximately 200 ELGANs annually. The standard of care in our unit is to treat extremely low birthweight infants $(<1000 \mathrm{~g})$ with prophylactic indomethacin $(0.1 \mathrm{mg} / \mathrm{kg}$ intravenous [IV] daily for 3 days), beginning within the first $6 \mathrm{~h}$ after birth.
\end{abstract}

\footnotetext{
${ }^{1}$ Department of Newborn and Developmental Pediatrics, Sunnybrook Health Sciences Centre, Toronto, ON, Canada. ${ }^{2}$ Department of Pediatrics, University of Toronto, Toronto, ON, Canada. ${ }^{3}$ Division of Neonatology, Department of Pediatrics, Hospital for Sick Children, Toronto, ON, Canada. ${ }^{4}$ Department of Neonatology, Fernandes Figueira Institute, Rio de Janeiro, Rio de Janeiro, Brazil. ${ }^{5}$ Department of Pediatrics, Mt. Sinai Hospital, Toronto, ON, Canada. ${ }^{\circledR}$ email: dany.weisz@sunnybrook.ca
}

Received: 14 May 2021 Revised: 9 July 2021 Accepted: 19 August 2021

Published online: 27 August 2021 
However, in 2010-2011, a prolonged shortage of indomethacin resulted in its administration being temporarily restricted to infants born at GA $\leq$ 25 weeks. Beyond the transitional period, PDA treatment was considered for neonates who developed a clinically symptomatic PDA (e.g. increasing ventilator support, cardiac murmur and/or wide pulse pressure) and sonographic evaluation was performed by a neonatologist with expertise in targeted neonatal echocardiography. Up to two courses of COXI therapy (indomethacin $[0.2 \mathrm{mg} / \mathrm{kg}$ intravenously every $12 \mathrm{~h}$ for three doses] or ibuprofen $[10 \mathrm{mg} / \mathrm{kg}$ intravenously/enterally once, followed by $5 \mathrm{mg} / \mathrm{kg}$ intravenously/enterally daily for two doses]) were administered to facilitate ductal closure among infants with clinically and echocardiographically significant PDA. The echocardiography criteria for large PDA included ductal diameter $\geq 2.0 \mathrm{~mm}$ and an unrestrictive systemic-topulmonary shunt [23], accompanied by echocardiography signs of left heart volume loading and pulmonary overcirculation (e.g. left atrium to aortic root ratio $\geq 1.5$, left ventricular dilatation, increased left ventricular output $[>300 \mathrm{ml} / \mathrm{kg} / \mathrm{min}]$ ) and/or diastolic flow reversal in the abdominal aorta. Echocardiography was performed using a Vivid E9 ultrasound machine (GE Healthcare, Chicago, USA) and a $12 \mathrm{MHz}$ phased array multifrequency probe.

Infants with persistent large PDA after unsuccessful pharmacological and/or conservative therapy and concomitant respiratory insufficiency (dependence on mechanical ventilation and/or high non-invasive mean airway pressure $\left[M A P \geq 8 \mathrm{~cm} \mathrm{H}_{2} \mathrm{O}\right.$ ] or fraction of inspired oxygen $\geq 0.3$ ) and/or end-organ hypoperfusion (e.g. renal insufficiency, inability to tolerate increases in enteral feeding volume) were considered candidates for surgical ligation [24, 25]. Infants were transported to our local quaternary centre for the procedure, which included a left lateral thoracotomy, and an intra- or extra-pleural approach using a clip or ligature at the discretion of the attending surgeon.

In July 2012, oral acetaminophen was introduced as a late therapy after failure or contraindication of COXI treatment among preterm infants with persistent large PDA being considered for surgical ligation. Acetaminophen treatment was administered with the goal of promoting sufficient ductal restriction to effect improvement in clinical stability and echocardiography indices of ductal shunting and avoid ligation.

To evaluate the association of late oral acetaminophen therapy vs. immediate surgical ligation with neonatal outcomes, we compared successive three-year cohorts of extremely preterm neonates who were considered candidates for PDA ligation. In the first epoch (July 1, 2009 to June 30,2012), infants were referred for PDA surgery directly after failure of COXI therapy [immediate surgical ligation epoch]. In the second epoch (July 1, 2012 to June 30, 2015), neonates with persistent large PDA were treated with a 7 day course of acetaminophen administered orally at a dose of $15 \mathrm{mg} / \mathrm{kg}$ every $6 \mathrm{~h}$ [acetaminophen epoch] [26]. Infants underwent echocardiography within 3 days of completing acetaminophen treatment and those with persistent clinical and echocardiography signs of large PDA after completion of the treatment course were referred for surgical ligation. Percutaneous transcatheter device closure for PDA among extremely preterm neonates was not available at our centre during either epoch.

\section{Outcomes and assessment}

Surviving infants underwent neurodevelopmental assessment at 18-24 months corrected age, which consisted of a clinical examination, visual and hearing assessment, and cognitive, language and motor evaluation using the Bayley Scales of Infant Development, Third Edition (BSID III). Clinical examinations and standardised motor assessments identified the presence of cerebral palsy, which was classified according to the Gross Motor Functional Classification System [27]. Standardised screening for autism spectrum disorder was performed and at-risk children were assessed using the Autism Diagnostic Observational Schedule.

We previously reported that late treatment with acetaminophen in this cohort was associated with reduced surgical ligation, but an increase in chronic lung disease [11]. In this follow-up study, we sought to evaluate the association of late acetaminophen treatment vs. immediate surgical ligation on clinical outcomes at 18-24 months corrected GA. The primary outcome was death or neurodevelopmental impairment. Neurodevelopmental impairment was defined as a composite of neuromotor, neurocognitive and/or neurosensory impairment. (Supplementary Table 1) Secondary outcomes (all estimated at 18-24 months corrected age) included death or severe NDI, mortality alone, NDI alone (any and severe), autism spectrum disorder, and PDA ligation.

\section{Data sources and collection}

Eligible infants were identified using local, comprehensive clinical and echocardiography databases and those with chromosomal abnormalities or congenital heart disease (other than secundum atrial septal defect or muscular ventricular septal defect) were excluded. Data on important antenatal and perinatal characteristics was abstracted from patient charts. To guide the appropriate collection of postnatal confounders, a date of study 'enrolment' was determined for each infant. Infants in both epochs were considered 'enroled' on the earliest date that they were considered a candidate for surgical ligation. The enrolment date for infants in the immediate surgical ligation epoch was the date of referral for PDA surgery. Infants in the acetaminophen epoch were considered enroled on the date of initiation of the course of acetaminophen. Detailed collection of postnatal, pre-enrolment characteristics was performed to enable adjustment for indices of illness severity and morbidities of prematurity that arose or were acquired prior to enrolment.

\section{Statistical analysis}

Descriptive statistics were used to summarise antenatal, perinatal and preenrolment characteristics for infants in the two epochs. Mean (standard deviation [SD]) and median (interquartile range [IQR]) were used to characterise normally distributed and skewed data, respectively. The characteristics of the infants in each epoch were compared using the Student's $t$ test or Wilcoxon rank-sum test for continuous variables and the chi-square test or Fisher's exact test for categorical variables, as appropriate. Multivariable logistic regression analysis was used to estimate the association between epoch and all outcomes, adjusting for perinatal and postnatal, pre-enrolment confounders. Covariates were included in the final model if associated with the primary outcome on univariate analysis $(p<0.20)$. (Supplementary Table 2 ) Goodness of fit was assessed using the Hosmer-Lemeshow statistic and all models were evaluated for collinearity and overspecification. Statistical analyses were performed using SAS version 9.4 (Cary, North Carolina). All statistical tests were two-sided with significance evaluated at the $5 \%$ level. This study was approved by the local hospital Research Ethics Board and was performed in accordance with the Declaration of Helsinki. Informed consent from the parents of study participants was waived due to the retrospective nature of the study.

\section{RESULTS}

From July 1, 2009 to June 30, 2015, 92 of 1034 infants born at $\leq 28$ weeks gestation had persistent large PDA and were considered candidates for surgical ligation after failure or contraindication to COXI and/or conservative therapy (43 of 467 [9.2\%] of infants in the immediate surgical ligation epoch and 49 of 567 [8.6\%] in the acetaminophen epoch) [Fig. 1]. The final analysis included 82 infants (38 in epoch 1 and 44 in epoch 2) for whom complete outcome information (death or neurodevelopmental impairment) was known, as five infants in each epoch were lost to neurodevelopmental follow-up. There were few significant differences between the acetaminophen and immediate surgical ligation groups in their antenatal and perinatal characteristics, except for known secular trends in clinical management (antenatal magnesium sulphate for foetal neuroprotection [50\% vs. $21 \%$, $p<0.01]$ and exclusively non-invasive delivery room respiratory support [ $23 \%$ vs. $5 \%, p=0.02]$ ). (Table 1 )

Infants in the acetaminophen group started COXI treatment later ( 10 vs. 6 days after birth, $p<0.01$ ), had a lower percentage of days of invasive mechanical ventilation at enrolment (93\% vs. $100 \%, p<0.01)$, and there were no differences in the age of PDA diagnosis, total dose of COXI treatment, or any other clinical characteristic. (Table 2) Infants treated with acetaminophen (40 of 49 infants in the acetaminophen epoch) received a median of 7 days of therapy (IQR: 7, 7) beginning at 26.5 days after birth (IQR: 21, 30.5; range 12-45). No infant in epoch 1 received acetaminophen treatment for PDA.

\section{Treatment with PDA ligation}

Compared with infants in the immediate surgical ligation group, infants in the acetaminophen group had decreased surgical 


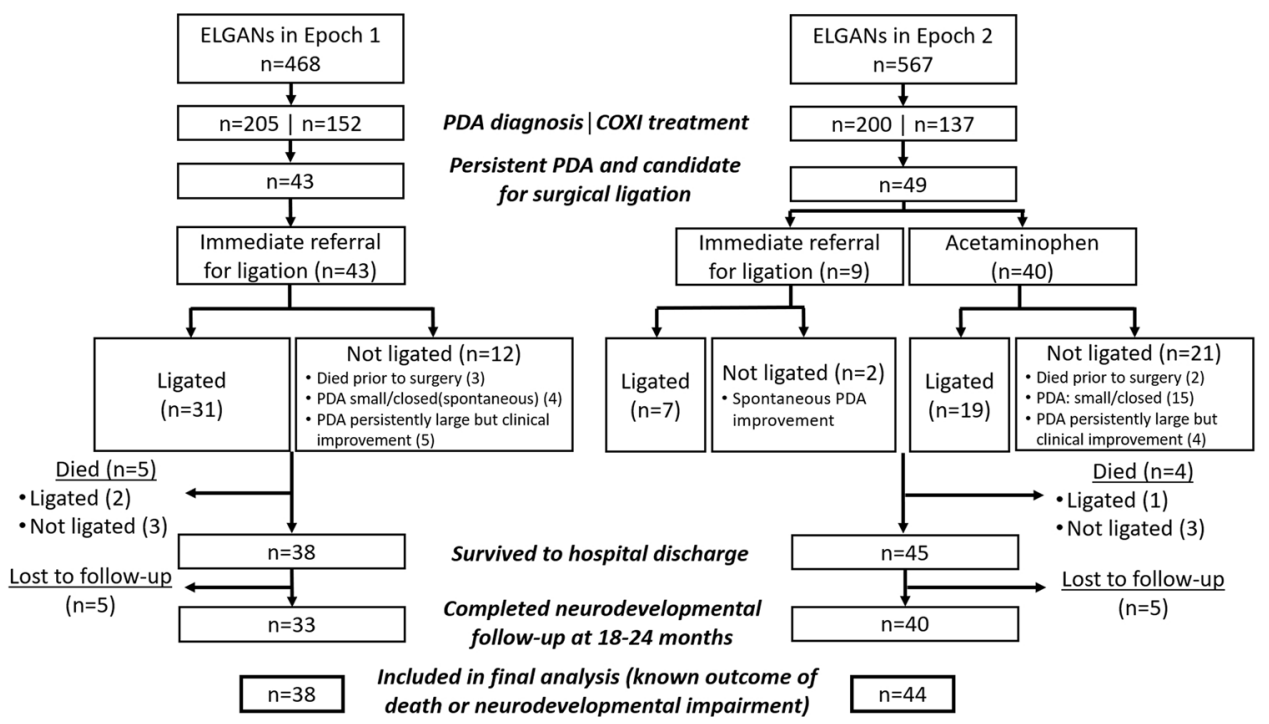

Fig. 1 Flow chart of infants included in the study. Forty-three of 468 extremely low gestational age neonates (ELGANs) in epoch 1 and 49 of 567 ELGANs in epoch 2 had persistent PDA and were candidates for surgical ligation. The primary outcome of death or neurodevelopmental impairment at 18-24 months was known for 38 (88\%) and 45 (92\%) of ELGANs in epochs 1 and 2, respectively.

Table 1. Antenatal and perinatal characteristics of preterm infants being considered for surgical ligation.

\begin{tabular}{|c|c|c|c|}
\hline & $\begin{array}{l}\text { Immediate surgical ligation } \\
\text { epoch }(n=38)\end{array}$ & Acetaminophen epoch $(n=44)$ & $p$ value \\
\hline Gestational age at birth (weeks) & $25.1(24.6,26.0)$ & $25.3(24.3,26.3)$ & 0.88 \\
\hline Small for gestational age ( $<10$ th percentile), $n$ (\%) & $4(11 \%)$ & $5(11 \%)$ & 0.90 \\
\hline Female sex, $n(\%)$ & $20(53 \%)$ & $24(55 \%)$ & 0.86 \\
\hline Antenatal corticosteroids, $n(\%)$ & $34(89 \%)$ & 39 (89\%) & 1.00 \\
\hline Magnesium sulfate for foetal neuroprotection, $n(\%)$ & $8(21 \%)$ & $22(50 \%)$ & $<0.01$ \\
\hline Caesarean delivery, $n$ (\%) & $26(68 \%)$ & $28(64 \%)$ & 0.65 \\
\hline Five-minute Apgar score & $7(6,8)$ & $7(5,7)$ & 0.27 \\
\hline Delivery room resuscitation & & & 0.02 \\
\hline Prophylactic indomethacin, $n$ (\%) & $14(37 \%)$ & $35(80 \%)$ & $<0.01$ \\
\hline Score for Neonatal Acute Physiology II & $14(14,27)$ & $19(9,25)$ & 0.95 \\
\hline
\end{tabular}

Data shown for infants for whom complete outcome information was available (death or neurodevelopmental impairment). Five infants in each group were lost to neurodevelopmental follow-up.

Continuous data presented as mean (SD) or median (IQR).

COXI cyclooxygenase inhibitors, CPAP continuous positive airway pressure, PDA patent ductus arteriosus.

ligation (53\% vs. $72 \%$, aOR $0.30,95 \%$ Cl: $0.11,0.87 ; p=0.03)$ (Table 3$)$. In epoch 1 , all infants $(n=43)$ were referred for ligation, though 12 did not ultimately undergo PDA surgery ( 3 infants died from sepsis; 4 infants experienced spontaneous ductal constriction prior to surgery; and for 5 infants, referral for ligation was cancelled in favour of conservative management due to clinical improvement in respiratory support [successful endotracheal extubation] despite a persistently large PDA on echocardiography). (Fig. 1) Among the 49 infants in epoch 2, 9 were immediately referred for PDA surgery (and did not receive acetaminophen treatment) owing to increased illness severity or contraindications for acetaminophen treatment, of whom 7 underwent ligation and 2 infants experienced spontaneous ductal constriction prior to PDA surgery. Among the 40 infants in epoch 2 who were treated with acetaminophen, 19 underwent PDA ligation after the treatment with acetaminophen was unsuccessful in facilitating ductal constriction. Among the 21 (out of 40) acetaminophen-treated infants who were not treated with ligation, 15 experienced significant improvement in ductal size and shunt volume (either PDA closure or reduction to a small shunt on echocardiography) while 4 experienced improvements in clinical respiratory stability only (without improvement in echocardiography indices of ductal 
Table 2. Clinical and echocardiography characteristics at enrolment and neonatal outcomes.

\begin{tabular}{|c|c|c|c|}
\hline & $\begin{array}{l}\text { Immediate } \\
\text { surgical ligation } \\
\text { epoch }(n=38)\end{array}$ & $\begin{array}{l}\text { Acetaminophen epoch } \\
(n=44)\end{array}$ & $p$ value \\
\hline \multicolumn{4}{|l|}{ Clinical characteristics } \\
\hline Enrolment weight $(\mathrm{g})$ & $970(846,1052)$ & $1004(822,1180)$ & 0.40 \\
\hline PDA initial diagnosis (day of life) & $4(4,6)$ & $5(3,7)$ & 0.94 \\
\hline Total COXI dose (including prophylactic indomethacin) $(\mathrm{mg} / \mathrm{kg})^{\mathrm{a}}$ & $1.2(1.2,1.5)$ & $1.45(0.75,1.5)$ & 0.83 \\
\hline Invasive ventilation at enrolment, $n(\%)$ & $34(89 \%)$ & $36(82 \%)$ & 0.33 \\
\hline Mean airway pressure at enrolment $\left(\mathrm{cmH}_{2} \mathrm{O}\right)$ & $10.9(3.1)$ & $11.1(3.0)$ & 0.68 \\
\hline Percentage of invasive ventilation days at enrolment & $100(95,100)$ & $93(70,100)$ & $<0.01$ \\
\hline Pulmonary haemorrhage, $n(\%)$ & $9(24 \%)$ & $7(16 \%)$ & 0.38 \\
\hline Sepsis, $n(\%)$ & $10(26 \%)$ & $15(34 \%)$ & 0.45 \\
\hline Systemic corticosteroids, $n$ (\%) & $2(5 \%)$ & $4(9 \%)$ & 0.51 \\
\hline \multicolumn{4}{|l|}{ Echocardiography characteristics } \\
\hline PDA diameter $(\mathrm{mm})$ & $2.4(2.2,2.8)$ & $2.6(2.2,3.2)$ & 0.05 \\
\hline Left ventricular output ( $\mathrm{ml} / \mathrm{kg} / \mathrm{min})$ & $426(130)$ & $404(74)$ & 0.43 \\
\hline Left atrium: Aortic root ratio & $1.9(1.6,2.2)$ & $2.0(1.8,2.2)$ & 0.56 \\
\hline \multicolumn{4}{|l|}{ Neonatal outcomes } \\
\hline Death before discharge, $n$ (\%) & $5(13 \%)$ & $4(9 \%)$ & 0.73 \\
\hline Moderate-severe bronchopulmonary dysplasia, $n(\%)^{b}$ & $22(65 \%)$ & $34(81 \%)$ & 0.11 \\
\hline Severe retinopathy of prematurity, $n(\%)^{c}$ & $4(12 \%)$ & $6(15 \%)$ & 0.72 \\
\hline
\end{tabular}

Data shown for infants for whom complete outcome information was available (death or neurodevelopmental impairment). Five infants in each group were lost to neurodevelopmental follow-up.

Continuous data presented as mean (SD) or median (IQR).

COXI cyclooxygenase inhibitors, CPAP continuous positive airway pressure, PDA patent ductus arteriosus.

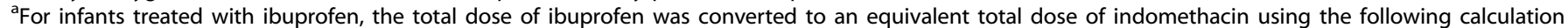

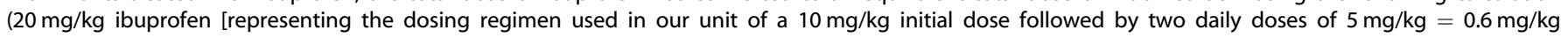
indomethacin [representing three doses of $0.2 \mathrm{mg} / \mathrm{kg}$ administered every $12 \mathrm{~h}$ ).

${ }^{\mathrm{b}}$ Moderate-severe bronchopulmonary dysplasia defined as the need for oxygen or positive pressure support at 36 weeks corrected gestational age.

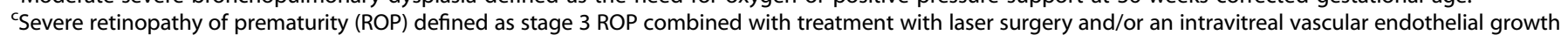
factor inhibitor.

size or shunt volume). (Fig. 1) Two acetaminophen-treated infants died prior to PDA ligation (one died from sepsis on the fifth day of acetaminophen treatment and the other died after withdrawal of life-sustaining intensive care due to intracranial injury). Surgically treated infants in the acetaminophen group underwent ligation at a later age compared with ligated infants in the earlier epoch (38 $[23,32]$ vs. $28[27,52]$ days after birth, $p<0.01)$ and had longer duration of exposure to large PDA (41 vs. 28 days, $p<0.01$ ). No infants underwent PDA ligation after discharge home from the NICU.

\section{Outcomes in early childhood}

There was no difference in the primary composite outcome of death or any NDI at $18-24$ months (70\% vs. $66 \%$; adjusted odds ratio (aOR) 1.03, 95\% confidence interval (Cl): 0.30, 3.56). (Table 3) There was no difference in any of the secondary outcomes, including death or severe NDI (45\% vs. $45 \%$; aOR $0.86,95 \% \mathrm{Cl}$ : $0.30,2.51)$, NDI alone (67\% vs. $61 \%$; aOR $1.02,95 \% \mathrm{Cl}: 0.28,3.65)$ or autism spectrum disorder ( $5 \%$ vs. $1 \%$, aOR $3.49,95 \% \mathrm{Cl}: 0.24$, 50.78; $p=0.36$ ).

The incidence of cerebral palsy was similar among both epochs (Epoch 1: $21 \%$ vs. Epoch 2: $13 \%, p=0.34$ ), as were the BSID III cognitive (median [IQR] $90[80,100]$ vs. $85[70,90], p=0.06)$, language $(79[65,94]$ vs. $77[60.5,90.5], p=0.54)$ and motor (88 $[82,97]$ vs. $85[73,94], p=0.17)$ scores.

Among infants in epoch 2 who were treated with acetaminophen $(n=40)$, there were no differences in early childhood outcomes among those treated vs. not treated with surgical ligation. (Supplementary Table 3).

\section{DISCUSSION}

In this retrospective comparative epoch study of ELGANs with persistent large PDA who were being considered for surgical ligation, treatment with a relatively prolonged course of oral acetaminophen was associated with decreased odds of PDA surgery and no difference in death or neurodevelopmental impairment in early childhood. These results suggest that late acetaminophen treatment may be effective and safe in facilitating PDA closure and avoiding surgical ligation without adverse neurodevelopmental effects in early childhood.

While treatment with acetaminophen has been demonstrated to be efficacious for facilitating ductal closure [10, 22, 28], few studies have evaluated the impact on neurodevelopmental 
Table 3. Outcomes in early childhood ${ }^{a}$.

\begin{tabular}{|c|c|c|c|c|c|}
\hline Outcome & $\begin{array}{l}\text { Immediate surgical } \\
\text { ligation epoch }\end{array}$ & Acetaminophen epoch & $\begin{array}{l}\text { Crude OR } \\
(95 \% \mathrm{Cl})\end{array}$ & $\begin{array}{l}\text { Adjusted OR } \\
(95 \% \mathrm{Cl})^{\mathrm{b}}\end{array}$ & $p$ value \\
\hline Death or Severe NDI, n/N (\%) & $17 / 38(45 \%)$ & $20 / 44(45 \%)$ & $1.03(0.43,2.46)$ & $0.86(0.30,2.51)$ & 0.78 \\
\hline Severe NDI, $n / N(\%)^{c}$ & $12 / 33(36 \%)$ & $16 / 40(40 \%)$ & $1.17(0.45,3.02)$ & $0.86(0.27,2.76)$ & 0.81 \\
\hline $\begin{array}{l}\text { Autism Spectrum Disorder, } \\
\mathrm{n} / \mathrm{N}(\%)^{\mathrm{c}}\end{array}$ & $1 / 33(1 \%)$ & $4 / 40(5 \%)$ & $3.55(0.38,33.5)$ & $3.49(0.24,50.78)$ & 0.36 \\
\hline $\begin{array}{l}\text { Ligation before } 18-24 \\
\text { months, n/N (\%) }\end{array}$ & $31 / 43(72 \%)$ & $26 / 49(53 \%)$ & $0.44(0.18,1.05)$ & $0.30(0.11,0.87)$ & 0.03 \\
\hline
\end{tabular}

Cl confidence interval, NDI neurodevelopmental impairment, OR odds ratio.

${ }^{a}$ Reference is the Immediate surgical ligation epoch (epoch 1). Ten infants (five infants per epoch) did not undergo neurodevelopmental evaluation and denominators for outcomes that include neurodevelopmental impairment include only infants for which complete outcomes were known.

${ }^{b}$ Adjusted for sex, outborn status, maternal exposure to magnesium sulfate for foetal neuroprotection, delivery room intubation, SNAP-II score, and the following postnatal confounders representing illness severity/acquired morbidity prior to or at the time of enrolment: treatment with inotropic drugs, culturenegative sepsis, culture-positive sepsis.

Included only infants who survived and completed neurodevelopmental follow-up (five infants died, and five infants did not complete a neurodevelopmental evaluation in the Immediate surgical ligation epoch; four infants died, and five infants did not complete a neurodevelopmental evaluation in the Acetaminophen epoch).

outcomes. Follow-up studies of pilot clinical trials of acetaminophen prophylaxis [20] (vs. placebo) and early treatment in the first 3 days after birth (vs. ibuprofen) [21] have reported no differences in neurodevelopmental impairment in early childhood. Knowledge of the long-term safety of acetaminophen for symptomatic PDA treatment is important as it is being increasingly prescribed by clinicians worldwide, who in contemporary practice may be seeking to avoiding treatment with surgical ligation and/or as an alternative to COXI [6-9]. Acetaminophen treatment among ELGANs with PDA in the Canadian Neonatal Network increased from $7 \%$ in 2014 to over $25 \%$ in 2019 [12, 29]. Similarly, in 2018, 1/ 3 of neonatal units in the United Kingdom administered acetaminophen for PDA treatment, mostly as second-line therapy after unsuccessful COXI therapy [30]. To the best of our knowledge, no prior studies have evaluated the long-term safety of later (beyond the first week after birth) or prolonged ( $>3$ days) courses of acetaminophen. In our study, longer courses (median 7 days) of acetaminophen treatment and a modestly prolonged exposure to large PDA (41 vs. 28 days) were not associated with NDI among ELGANs with persistent PDA compared with immediate referral for surgical ligation.

Over the past decade, there have been concerns raised that prenatal or neonatal exposure, or both, to acetaminophen may adversely affect cerebral development. Prenatal exposure to acetaminophen has been associated with language delay, lower intelligence quotient, autism spectrum disorder and ADHD in childhood [31-34]. Longer duration of prenatal exposure has been related to increased risk, even after adjusting for indications for use, familial risk and other confounders [34]. Neonatal exposure to acetaminophen has similarly been associated with behaviour and cognitive deficits in a murine model [35]. In addition, increased autism spectrum disorder was associated with neonatal acetaminophen exposure in a human ecological study of country-specific rates of neonatal circumcision [31].

Several potential pathobiological mechanisms may explain the association of acetaminophen exposure and neurodevelopmental disorders. Acetaminophen may interrupt brain development by induction of oxidative stress, leading to neuronal death [36]. Prenatal exposure to acetaminophen has been associated, in a dose-dependent relationship, with increased DNA methylation among children with ADHD [37]. The genes affected by DNA methylation were largely those enriched in pathways involved in oxidative stress and neurological function [37]. In addition, acetaminophen may interfere with maternal and neonatal hormones (e.g. thyroid) related to brain development [38], and alter the expression of brain-derived neurotrophic factor, impacting neuronal growth, migration, and survival, and resulting in altered behaviour and reduced learning abilities [35, 39]. Although clinical toxicity has not been reported among ELGANs treated with acetaminophen for PDA, a recent review identified 22 case reports of acetaminophen-related hepatotoxicity in neonates when administered for other indications using similar dosing regimens [40]. In addition, studies of single administrations of high-dose acetaminophen have reported detectable toxic metabolites, indicating the potential for toxicity and stressing the need for careful and rigorous monitoring among ELGANs receiving repeated and prolonged courses of high-dose acetaminophen [41].

The strengths of this study include the in-depth abstraction of postnatal morbidities and indices of illness severity, such as daily respiratory support. These data collection included key covariates in the multivariable analyses to minimise confounding by indication. In addition, the proportion of infants lost to neurodevelopmental follow-up was low (10.9\%) [42].

Our study has several limitations; first, as a comparative epoch cohort study, improvements in clinical care and outcomes over time may be misattributed to the exposure under investigation (i.e. acetaminophen vs. surgical ligation treatment). In addition, the secular trend toward more selective PDA treatment may have resulted in selection bias in assembling the cohort of infants in each epoch considered eligible for ligation. However, the similar proportion of eligible infants in each epoch (9.2\% vs. $8.6 \%$ ) may suggest against the possibility of significant selection bias. Further, our inclusion of infants in epoch 2 who were not treated with acetaminophen (and instead immediately referred for ligation) may also have mitigated the potential for selection bias, as similar infants considered ineligible for acetaminophen treatment may have been included in epoch 1. Second, the observational nature of this study means that the true causal effect of late acetaminophen treatment in avoiding surgical ligation, compared with the potential for spontaneous closure over time, cannot be definitively established. Third, neurodevelopmental follow-up occurred too early in childhood (18-24 months corrected gestational age) to permit the appropriate evaluation and diagnosis of ADHD [43], which is the disorder that has been most 
strongly associated with acetaminophen exposure. Fourth, this study was underpowered to detect clinically important differences in the frequency of major, but uncommon, neurodevelopmental disorders such as ASD. Finally, 18-24 month neurodevelopmental evaluation of children born preterm may have limited reliability compared with outcomes later in childhood [44]. Additional, wellpowered studies are required to evaluate the association of acetaminophen treatment for PDA among preterm infants with neurodevelopmental outcomes at school-age.

\section{CONCLUSION}

The clinical approach of administering late treatment with oral acetaminophen among extremely preterm infants with persistent large PDA was associated with reduced surgical ligation and no difference in death or neurodevelopmental impairment in early childhood. These results support the safety and effectiveness of late acetaminophen treatment for PDA to avoid surgical ligation.

\section{REFERENCES}

1. Sellmer A, Bjerre JV, Schmidt MR, McNamara PJ, Hjortdal VE, Host B, et al. Morbidity and mortality in preterm neonates with patent ductus arteriosus on day 3 . Arch Dis Child Fetal Neonatal Ed. 2013;98:F505-10.

2. Noori S, McCoy M, Friedlich P, Bright B, Gottipati V, Seri I, et al. Failure of ductus arteriosus closure is associated with increased mortality in preterm infants. Pediatrics. 2009;123:e138-44.

3. Kabra NS, Schmidt B, Roberts RS, Doyle LW, Papile L, Fanaroff A, et al. Neurosensory impairment after surgical closure of patent ductus arteriosus in extremely low birth weight infants: results from the trial of indomethacin prophylaxis in preterms. J Pediatr. 2007;150:229-34.

4. Madan JC, Kendrick D, Hagadorn Jl, Frantz ID 3rd. Patent ductus arteriosus therapy: impact on neonatal and 18-month outcome. Pediatrics. 2009:123:674-81.

5. Weisz DE, More K, McNamara PJ, Shah PS. PDA ligation and health outcomes: a meta-analysis. Pediatrics. 2014;133:e1024-46.

6. Lokku A, Mirea L, Lee SK, Shah PS.Canadian Neonatal Network Trends and outcomes of patent ductus arteriosus treatment in very preterm infants in Canada. Am J Perinatol.2017;34:441-50.

7. Bixler GM, Powers GC, Clark RH, Walker MW, Tolia VN. Changes in the diagnosis and management of patent ductus arteriosus from 2006 to 2015 in United States neonatal intensive care units. J Pediatr. 2017;189:105-12.

8. Hagadorn Jl, Brownell EA, Trzaski JM, Johnson KR, Lainwala S, Campbell BT, et al. Trends and variation in management and outcomes of very low-birth-weight infants with patent ductus arteriosus. Pediatr Res. 2016;80:785-92.

9. Ngo S, Profit J, Gould JB, Lee HC. Trends in patent ductus arteriosus diagnosis and management for very low birth weight infants. Pediatrics. 2017;139:e20162390.

10. Kluckow $M$, Carlisle $H$, Broom $M$, Woods $P$, Jeffery $M$, Desai $D$, et al. A pilot randomised blinded placebo-controlled trial of paracetamol for later treatment of a patent ductus arteriosus. J Perinatol. 2019;39:102-7.

11. Mashally S, Nield LE, McNamara PJ, Martins FF, El-Khuffash A, Jain A, et al. Late oral acetaminophen versus immediate surgical ligation in preterm infants with persistent large patent ductus arteriosus. J Thorac Cardiovasc Surg. 2018;156:1937-44.

12. 2019 Canadian Neonatal Network Annual Report. Publication year: 2020. http:// www.canadianneonatalnetwork.org/portal/. Accessed 2 Jan 2021.

13. Weisz DE, Mirea L, Resende MHF, Ly L, Church PT, Kelly E, et al. Outcomes of surgical ligation after unsuccessful pharmacotherapy for patent ductus arteriosus in neonates born extremely preterm. J Pediatr. 2018;195:292-6 e3.

14. Weisz DE, Mirea L, Rosenberg E, Jang M, Ly L, Church PT, et al. Association of patent ductus arteriosus ligation with death or neurodevelopmental impairment among extremely preterm infants. JAMA Pediatr. 2017;171:443-9.

15. Liew Z, Ritz B, Virk J, Olsen J. Maternal use of acetaminophen during pregnancy and risk of autism spectrum disorders in childhood: a Danish national birth cohort study. Autism Res. 2016;9:951-8.

16. Ji Y, Riley AW, Lee LC, Hong X, Wang G, Tsai HJ, et al. Maternal biomarkers of acetaminophen use and offspring attention deficit hyperactivity disorder. Brain Sci. 2018;8:127.

17. Baker BH, Lugo-Candelas $\mathrm{C}$, Wu H, Laue HE, Boivin A, Gillet V, et al. Association of prenatal acetaminophen exposure measured in meconium with risk of attentiondeficit/hyperactivity disorder mediated by frontoparietal network brain connectivity. JAMA Pediatr. 2020;174:1-9.
18. Ji Y, Azuine RE, Zhang Y, Hou W, Hong X, Wang G, et al. Association of cord plasma biomarkers of in utero acetaminophen exposure with risk of attentiondeficit/hyperactivity disorder and autism spectrum disorder in childhood. JAMA Psychiatry. 2020;77:180-9.

19. Juujärvi S, Saarela T, Pokka T, Hallman M, Aikio O. Intravenous paracetamol for neonates: long-term diseases not escalated during 5 years of follow-up. Arch Dis Child Fetal Neonatal Ed. 2021;106:178-83.

20. Juujärvi S, Kallankari H, Pätsi $P$, Leskinen $M$, Saarela T, Hallman M, et al. Follow-up study of the early, randomised paracetamol trial to preterm infants, found no adverse reactions at the two-years corrected age. Acta Paediatr. 2019;108:452-8.

21. Oncel MY, Eras Z, Uras N, Canpolat FE, Erdeve O, Oguz SS. Neurodevelopmental outcomes of preterm infants treated with oral paracetamol versus ibuprofen for patent ductus arteriosus. Am J Perinatol. 2017;34:1185-9.

22. Ohlsson A, Shah PS. Paracetamol (acetaminophen) for patent ductus arteriosus in preterm or low birth weight infants. Cochrane Database Syst Rev. 2020;1: Cd010061.

23. Smith A, Maguire M, Livingstone V, Dempsey EM. Peak systolic to end diastolic flow velocity ratio is associated with ductal patency in infants below 32 weeks of gestation. Arch Dis Child Fetal Neonatal Ed. 2015;100:F132-6.

24. Resende MH, More K, Nicholls D, Ting J, Jain A, McNamara PJ. The impact of a dedicated patent ductus arteriosus ligation team on neonatal health-care outcomes. J Perinatol. 2016;36:463-8.

25. McNamara PJ, Sehgal A. Towards rational management of the patent ductus arteriosus: the need for disease staging. Arch Dis Child Fetal Neonatal Ed. 2007;92:F424-7.

26. Weisz DE, Martins FF, Nield LE, El-Khuffash A, Jain A, McNamara PJ. Acetaminophen to avoid surgical ligation in extremely low gestational age neonates with persistent hemodynamically significant patent ductus arteriosus. J Perinatol. 2016;36:649-53.

27. Palisano R, Rosenbaum P, Walter S, Russell D, Wood E, Galuppi B. Development and reliability of a system to classify gross motor function in children with cerebral palsy. Dev Med Child Neurol 1997;39:214-23.

28. Schindler T, Smyth J, Bolisetty S, Michalowski J, Mallitt KA, Singla A, et al. Early PARacetamol (EPAR.) trial: a randomized controlled trial of early paracetamol to promote closure of the ductus arteriosus in preterm infants. Neonatology. 2021;8:274-281.

29. 2014 Canadian Neonatal Network Annual Report Publication year: 2015. http:// www.canadianneonatalnetwork.org/portal/. Accessed 2 Jan 2021.

30. Noureldein M, Hu K, Groucutt J, Heaver R, Gurusamy K. Paracetamol for patent ductus arteriosus in preterm infants: a UK national survey. J Matern Fetal Neonatal Med. 2020:1-4. online ahead of print.

31. Bauer AZ, Kriebel D. Prenatal and perinatal analgesic exposure and autism: an ecological link. Environ Health. 2013;12:41.

32. Bauer AZ, Kriebel D, Herbert MR, Bornehag CG, Swan SH. Prenatal paracetamol exposure and child neurodevelopment: a review. Horm Behav. 2018;101:125-47.

33. Bornehag CG, Reichenberg A, Hallerback MU, Wikstrom S, Koch HM, Jonsson BA, et al. Prenatal exposure to acetaminophen and children's language development at 30 months. Eur Psychiatry. 2018;51:98-103.

34. Ystrom E, Gustavson K, Brandlistuen RE, Knudsen GP, Magnus P, Susser E, et al. Prenatal exposure to acetaminophen and risk of ADHD. Pediatrics. 2017;140: e20163840.

35. Viberg H, Eriksson P, Gordh T, Fredriksson A. Paracetamol (acetaminophen) administration during neonatal brain development affects cognitive function and alters its analgesic and anxiolytic response in adult male mice. Toxicol Sci. 2014;138:139-47.

36. Posadas I, Santos P, Blanco A, Munoz-Fernandez M, Cena V. Acetaminophen induces apoptosis in rat cortical neurons. PLoS One. 2010;5:e15360.

37. Gervin K, Nordeng H, Ystrom E, Reichborn-Kjennerud T, Lyle R. Long-term prenatal exposure to paracetamol is associated with DNA methylation differences in children diagnosed with ADHD. Clin Epigenetics. 2017;9:77.

38. Ghassabian A, Bongers-Schokking JJ, Henrichs J, Jaddoe VW, Visser TJ, Visser W, et al. Maternal thyroid function during pregnancy and behavioral problems in the offspring: the generation R study. Pediatr Res. 2011;69:454-9. 5 Pt 1

39. Huang EJ, Reichardt LF. Neurotrophins: roles in neuronal development and function. Annu Rev Neurosci. 2001;24:677-736.

40. Locci C, Cuzzolin L, Capobianco G, Antonucci R. Paracetamol overdose in the newborn and infant: a life-threatening event. Eur J Clin Pharmacol. 2021;77:809-15.

41. Flint RB, Roofthooft DW, van Rongen $A$, van Lingen RA, van den Anker JN, van Dijk $M$, et al. Exposure to acetaminophen and all its metabolites upon 10,15, and $20 \mathrm{mg} / \mathrm{kg}$ intravenous acetaminophen in very-preterm infants. Pediatr Res. 2017;82:678-84.

42. Kristman V, Manno M, Cote P. Loss to follow-up in cohort studies: how much is too much? Eur J Epidemiol. 2004;19:751-60. 
43. Loe IM, Kakar PA, Sanders LM. Diagnosis, evaluation, and treatment of attentiondeficit/hyperactivity disorder. JAMA Pediatr. 2021;175:191-2.

44. Taylor GL, Joseph RM, Kuban KCK, Douglass LM, Laux J, Andrews B, et al. Changes in neurodevelopmental outcomes from age 2 to 10 years for children born extremely preterm. Pediatrics. 2021;147:e2020001040.

\section{AUTHOR CONTRIBUTIONS}

SM contributed to study design, acquisition and interpretation of data, reviewed and revised the manuscript, and approved the final manuscript. RB, BJ, LN, FM and AJ contributed to study design, interpretation of data, reviewed and revised the manuscript, and approved the final manuscript. DW contributed to study design, the acquisition, analysis and interpretation of study data, wrote the first draft of the manuscript, reviewed and revised the manuscript, and approved the final manuscript.

\section{COMPETING INTERESTS}

The authors declare no competing interests.

\section{ADDITIONAL INFORMATION}

Supplementary information The online version contains supplementary material available at https://doi.org/10.1038/s41372-021-01194-4.

Correspondence and requests for materials should be addressed to D.E.W.

Reprints and permission information is available at http://www.nature.com/ reprints

Publisher's note Springer Nature remains neutral with regard to jurisdictional claims in published maps and institutional affiliations. 